\title{
Comment on Doppler Ultrasound in Vascular Access care: The pearls and pitfalls of flow volume measurements
}

\author{
Cora de Bont ${ }^{1}$, Jan Malik², Kirk Beach ${ }^{3}$ \\ ${ }^{1}$ Vascular Laboratory, Bravis Hospital, Bergen op Zoom, the Netherlands \\ ${ }^{2}$ Center for Vascular Access, General University Hospital, First Faculty of Medicine, Charles University, Prague, Czech Republic \\ ${ }^{3}$ Departments of Surgery and Bioengineering, University of Washington, Seattle, WA, USA
}

We have read the recent review of Diniz and Coentrão ${ }^{1}$ entitled "Doppler Ultrasound in Vascular Access care: the pearls and pitfalls of flow volume measurements" with a great interest.

The authors mention that Doppler ultrasound (DUS) is an operator dependent technique that needs substantial time of learning. This ultrasound learning curve will probably not be longer than for any other imaging method. The problem rather is that ultrasound looks (too) easy. Studies proving that DUS is reliable will be most applicable to situations where the examinations were performed by experienced (vascular) ultrasonographists. ${ }^{2}$

Even by offering handles and pointing out potential errors, which is a good thing, mistakes by less experienced operators are easily made. Probably unintentionally and unfortunately the authors themselves made some mistakes in their included image like the use of a Doppler angle $>60^{\circ}$ (Figure 1).

Ultrasound devices offer one or two time velocity integrals: the time-averaged maximum velocity (TAMAX), which is the time integral of the fastest velocity layers and/or the time-averaged mean velocity (TAMV) which is the time integral of the average of the velocity layers. Using TAMAX for calculating the flow volume of an arteriovenous access (Qa) will significantly overestimate $Q$ a. The device used by the authors, although it uses the abbreviation TAMV seems to measure
TAMAX. We roughly estimated that the real TAMV will be around 43 $\mathrm{cm} / \mathrm{s}$ (Figure 1), using this TAMV Qa becomes $<500 \mathrm{ml} / \mathrm{min}$.

The authors conclude that clinicians should keep in mind that Qa measurements with DUS are usually lower than determined with ultradilution. We think this conclusion has a fragile questionable base. Observed differences could depend on the used DUS machine. ${ }^{3} \mathrm{Qa}$ also depends on variables like the cardiac output, mean arterial pressure and total blood volume, so when comparing it is important to measure Qa at the same time. And although the brachial artery is recommended for the determination of Qa, Robbin et al. ${ }^{4}$ found that Qa measured in the vein represents the status of the fistula better. This will be most related, in our opinion, to the presence of multiple outflow veins. Qa measured with ultradilution in one of these outflow veins will be lower than Qa measured with DUS in the brachial artery.

The included DUS criteria for defining a significant stenosis were made up by Malik et al. ${ }^{5}$ These criteria are based on the so called "Watch and observe strategy". Treat as late as possible, but act before the access occludes. The authors mention that research showing the benefit for periodic surveillance of an arteriovenous graft (AVG) is missing. Striking fact, the only randomised trial that proved the benefit of periodic surveillance of AVGs with DUS, used the proposed DUS criteria. ${ }^{5}$

\section{Disclosure of potential conflicts of interest: none declared}

\section{Figure 1}

Flow volume determination with Doppler ultrasound.

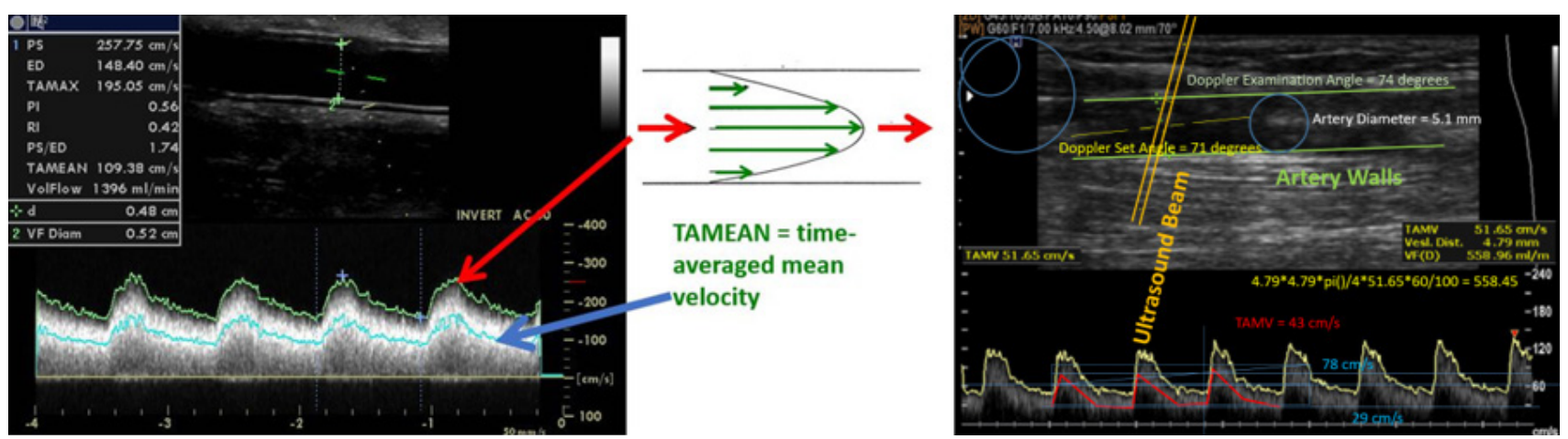

The left side points out the significant difference between TAMAX and TAMV, the right side is the altered figure of the authors (used with permission of Hugo Diniz). In it a rough estimation of the real TAMV and used Doppler angle $\left(>60^{\circ}\right)$. 


\section{References}

1. Diniz H, Coentrão L. Doppler ultrasound in vascular access care: the pearls and pitfalls of flow volume measurement. Port J Nephrol Hypert 2020; 34(1):25-30.

2. Zonnebeld N, Maas TMG, Huberts W et al. Pre-operative duplex ultrasonography in arteriovenous fistula creation: intra- and inter-observer agreement. Eur J Vasc Endovasc Surg. 2017 Nov; 54(5): 613-9.

3. Hoyt K, Hester FA, Bell RL et al. Accuracy of volumetric flow rate measurements an in vitro study using modern ultrasound scanners. J Ultrasound Med. 2009 Nov; 28(11):1511-8.
4. Robbin ML, Greene T, Allon M et al. Prediction of arteriovenous fistula clinical maturation from postoperative ultrasound measurements: findings from the hemodialysis fistula maturation study. J Am Soc Nephrol. 2018 Nov; 29(11):2735-44.

5. Malik J, Slavikova M, Svobodova J et al. Regular ultrasonographic screening significantly prolongs patency of PTFE grafts. Kidney Int. 2005 Apr;67(4):1554-8.

\section{Correspondence to:}

Cora de Bont

E-mail: c.vanderheijden@bravis.nl 